\title{
LHC observables with NNLOJET
}

\author{
Rhorry Gauld \\ Nikhef Theory Group, Science Park 105, 1098 XG Amsterdam, The Netherlands \\ E-mail: rgauldenikhef.nl
}

\section{Aude Gehrmann-De Ridder*}

Institute for Theoretical Physics, ETH, CH-8093 Zürich,

Physics Institute, University of Zürich, Winterthurerstrasse 190, CH-8057, Zürich, Switzerland

E-mail: gehra@phys.ethz.ch

\section{Nigel Glover}

Institute for Particle Physics Phenomenology, Department of Physics, University of Durham, Durham, DH1 3LE, UK

E-mail: e.w.n.gloveredurham.ac.uk

\section{Alexander Huss}

Theoretical Physics Department, CH-1211 Geneve 23, Switzerland

E-mail: alexander.hussecern.ch

\section{Imre Majer}

Institute for Theoretical Physics, ETH, CH-8093 Zürich, Switzerland

E-mail:majeriephys.ethz.ch

\begin{abstract}
We report the main features of the computation of the NNLO corrections to the Higgs Strahlung processes $\mathrm{W}^{+} \mathrm{H}, \mathrm{W}^{-} \mathrm{H}$, and $\mathrm{ZH}$ including the off-shell leptonic decay of the gauge boson as well as the Higgs decaying into a bottom-antibottom pair. The calculation consistently takes into account NNLO QCD corrections to the production and decay sub-processes and fully retains the differential information on the final state.
\end{abstract}

14th International Symposium on Radiative Corrections (RADCOR2019)

9-13 September 2019

Palais des Papes, Avignon, France

\footnotetext{
* Speaker.
} 


\section{Introduction}

The production of a Higgs boson $(\mathrm{H})$ in association with either a $\mathrm{W}^{ \pm}$or a $\mathrm{Z}$ boson and possible hadronic jets - also known as Higgs Strahlung — is among the most promising class of channels that can lead to the accurate determination of the Higgs-boson couplings. In particular, it offers the possibility to study the decay of a Higgs boson into a pair of bottom-antibottom quarks. Such a decay is hard to measure in inclusive Higgs production through the leading production modes like the gluon-gluon or vector-boson-fusion channels due to the presence of enormous QCD backgrounds. In the Higgs Strahlung process the presence of a vector boson decaying leptonically provides a clean experimental signature and experimental analyses related to $V \mathrm{H}$ production have manageable backgrounds.

In 2017, the LHC experiments [1,2] announced the observation of a SM Higgs-like particle decaying to a pair of bottom-antibottom quarks precisely through this Higgs Strahlung production channel with a significance of 5.6 and 5.3 standard deviations for CMS and ATLAS respectively. Most recently, the ATLAS collaboration reported first differential measurements considering a simplified template cross section as a function of the transverse momentum of the vector boson, [3].

In view of prospective measurements of Higgs Strahlung final states including data from Run II and III at the LHC, it is of crucial importance to have precise theoretical predictions for cross sections and differential distributions in the kinematic regions probed by the experiments. This includes in particular QCD effects in both the production and in the decay of the Higgs boson into a bottom-quark pair and critically relies on the tagging of bottom jets in order to isolate the candidate pairs associated to the Higgs boson. It is the aim of this report to present a computation of $V \mathrm{H}$ observables for all three processes $\left(V=\mathrm{Z}, \mathrm{W}^{ \pm}\right)$including NNLO corrections to both production and decay sub-processes retaining a fully differential description of the final state including offshell propagators of the Higgs and vector boson. Those results have been described in [4].

In this talk, we first describe how flavour-dependent observables are computed at fixed-order accuracy within the parton level event generator NNLOJET before presenting the different ingredients appearing in production and decay sub-processes up to NNLO level of the associated Higgs production processes. Results for fiducial cross sections and distributions are then presented for the LHC at $\sqrt{s}=13 \mathrm{TeV}$. Those include, for the first time, scale uncertainty estimations related to the separate variation of production and decay scales at each order in $\alpha_{\mathrm{s}}$.

Besides this computation fully differential NNLO predictions for $V H$ observables obtained via the combination of Higgs production and decay to bottom-antibottom processes have been presented in ref. [5] (for $V=\mathrm{Z}, \mathrm{W}^{+}$) and in ref. [6] (for $V=\mathrm{W}^{-}$). Unlike the computation presented here and in [4], a scaling procedure to a fixed branching ratio is used in these aforementioned computations to define the cross section expressions at each perturbative order. By explicitly comparing the fiducial cross sections in these two frameworks, we argue furthermore in this work, that starting from NNLO the latter scaling procedure could be inadequate in estimating missing higher-order effects through scale variations.

\section{Flavour sensitive jet observables at NNLO}

The presence of two identified b-jets with a combined invariant mass consistent with $m_{\mathrm{H}}$ al- 
lows us to associate this final state with the underlying process $\mathrm{pp} \rightarrow V \mathrm{H}+X \rightarrow \ell \bar{\ell} \mathrm{b} \overline{\mathrm{b}}+X$. The identification of jet flavour is an essential component of any experimental analysis of this process, which is required to reduce otherwise overwhelming background processes. It is therefore also an integral part of the requirements needed to obtain the corresponding theoretical predictions.

The computation of such observables at fixed order requires the application of a flavoursensitive jet algorithm that - besides reconstructing flavour-insensitive properties such as fourmomenta - identifies the flavour of the reconstructed jets based on some well-defined (infraredsafe) criteria [7]. The application of such an algorithm which will be given below requires a tracking of the flavour of individual partons, which appear in the partonic cross section at each perturbative order beforehand. In the following, we provide a description of how this is achieved within the parton-level event generator NNLOJET. It is worth mentioning, that the following flavour tagging procedure is general: it is not restricted to the $\mathrm{VH}$ processes with the Higgs boson decaying into flavoured b-jets but could be applied on top of any existing flavour-blind computations already present in the NNLOJET framework to generate flavour sensitive observables.

\subsection{Flavour dressing in NNLOJET}

The first step towards computing flavour-dependent jet observables is to ensure that the jet algorithm has access to both momentum and flavour information when evaluating the contributions from matrix elements and subtraction terms. To address this issue within NNLOJET, an additional "flavour-dressing" layer that tracks the flavours of all amplitudes as well as reduced matrix elements appearing in subtraction terms has been implemented.

As an example of the flavour-dressing procedure for the amplitudes, we consider in a generic NLO-type cross section for an $n$-body final state initiated by the two partons $i$ and $j$, the construction of the real-emission cross section (omitting the sum over potential colour orderings). Following the notation of ref. [8], it reads:

$$
\begin{aligned}
\mathrm{d} \hat{\sigma}_{i j, \mathrm{NLO}}^{R} & =\mathscr{N}_{\mathrm{NLO}}^{R} \mathrm{~d} \Phi_{n+1}\left(\left\{p_{3}, \ldots, p_{n+3}\right\} ; p_{1}, p_{2}\right) \frac{1}{S_{n+1}} \\
& \times\left[M_{n+3}^{0}\left(\left\{p_{n+3}\right\},\left\{f_{n+3}\right\}\right) J_{n}^{(n+1)}\left(\left\{p_{n+1}\right\},\left\{f_{n+1}\right\}\right)\right] .
\end{aligned}
$$

In this equation, we denote the final-state symmetry factor by $S_{n+1}$, the normalisation factor (which includes constants, couplings, colour factors) by $\mathscr{N}_{\mathrm{NLO}}^{R}$, the $2 \rightarrow n+1$ particle phase space by $\mathrm{d} \Phi_{n+1}$, and the momentum of the partons $i, j$ by $p_{1,2}$. The partial squared amplitude $M_{n+3}^{0}$ is evaluated with the momentum set $\left\{p_{n+3}\right\}$ and a corresponding flavour set $\left\{f_{n+3}\right\}$. The flavoursensitive jet algorithm $J_{n}^{(n+1)}$ builds $n$ jets from $n+1$ final-state partons which carry momentum and flavour labelled by the sets $\left\{p_{n+1}\right\}$ and $\left\{f_{n+1}\right\}$ respectively.

The real subtraction cross section can be written in a similar fashion:

$$
\begin{aligned}
\mathrm{d} \hat{\sigma}_{i j, \mathrm{NLO}}^{S} & =\mathscr{N}_{\mathrm{NLO}}^{R} \sum_{k} \mathrm{~d} \Phi_{n+1}\left(\left\{p_{3}, \ldots, p_{n+3}\right\} ; p_{1}, p_{2}\right) \frac{1}{S_{n+1}} \\
& \times\left[X_{3}^{0}(\cdot, k, \cdot) M_{n+2}^{0}\left(\left\{\tilde{p}_{n+2}\right\},\left\{\tilde{f}_{n+2}\right\}\right) J_{n}^{(n)}\left(\left\{\tilde{p}_{n}\right\},\left\{\tilde{f}_{n}\right\}\right)\right],
\end{aligned}
$$

where the index $k$ runs over all possible unresolved partons in $\mathrm{d} \hat{\sigma}_{i j \text {,NLO }}^{R}$ and $X_{3}^{0}(\cdot, k, \cdot)$ denotes the three-parton antenna function that factorises from the associated reduced squared matrix-element 
$M_{n+2}^{0}$. In this case, the jet algorithm acts upon mapped final-state momentum and flavour sets $\left\{\tilde{p}_{n}\right\}$ and $\left\{\tilde{f}_{n}\right\}$ associated with the reduced squared matrix element $M_{n+2}^{0}$. As the total subtraction cross section must take into account all possible unresolved limits of parton $k$, this cross section may be composed of multiple flavour structures. The subtraction method is only effective if the evaluation of flavour-dependent observables in both the real and real-subtraction cross sections match in all possible unresolved limits. This is only ensured if an infrared-safe flavour-sensitive jet algorithm is applied.

To construct the NLO cross section, a similar procedure must also be applied to both virtual and virtual-subtraction (in the antenna formalism, these include integrated subtraction and massfactorisation contributions) cross sections. To allow the computation of flavour-dependent jet observables at NNLO, the same ideas extend to one order higher and this flavour-dressing procedure is applied to all NNLO-type parton level contributions and their corresponding subtraction terms.

\subsection{Flavour-aware jet algorithm}

Throughout this work jets are reconstructed with the flavour- $k_{t}$ algorithm, which provides an infrared-safe definition of jet flavour. The main difference with respect to a native jet algorithm is that the clustering of particles relies on both momentum and flavour information of the input pseudo-jets. The essential steps of the algorithm for hadron-hadron collisions originally presented in ref. [7] (also summarised in ref. [9]) can be sketched as follows:

The algorithm proceeds by assigning a net flavour to all pseudo-jets or jets based on their quark flavour content, attributing $+1(-1)$ if a quark (antiquark) of the flavour under consideration is present. In an experimental context, the presence of a quark flavour could be inferred from a fully/partially reconstructed hadron. A criterion is then applied to these objects to determine if they carry flavour, possible examples being: the net flavour (sum of quarks and antiquarks); or the net flavour modulo two. Objects are considered to carry flavour if they carry non-zero values of this criterion. The algorithm then proceeds by constructing distance measures for pairs of all final-state pseudo-jets $i$ and $j\left(d_{i j}\right)$ as well as beam distances $\left(d_{i B}\right.$ and $\left.d_{i \bar{B}}\right)$ as described explicitly in [4] . In particular, the (flavour-dependent) distance $\left(d_{i j}\right)$ for all final-state pseudo-jets $i$ and $j$ are defined as

$$
d_{i j}=\frac{\Delta y_{i j}^{2}+\Delta \phi_{i j}^{2}}{R^{2}} \begin{cases}\max \left(k_{t i}, k_{t j}\right)^{\alpha} \min \left(k_{t i}, k_{t j}\right)^{2-\alpha} & \text { softer of } i, j \text { is flavoured, } \\ \min \left(k_{t i}, k_{t j}\right)^{\alpha} & \text { softer of } i, j \text { is unflavoured, }\end{cases}
$$

In these definitions, $k_{t i}$ and $k_{t j}$ are the transverse momentum of the pseudo-jets $i$ and $j$, and the rapidity difference and azimuthal angular separation between these pseudo-jets is given by $\Delta y_{i j}$ and $\Delta \phi_{i j}$, respectively. The parameters $R$ and $\alpha$ define a class of measures for the algorithm.

While this flavour-aware jet algorithm is substantially more complex than the flavour-blind anti- $k_{t}$ algorithm [10], its use is unavoidable in fixed-order computations based on massless quarks. At NNLO in particular, the flavour-dependent distance measure in eq. (2.3) ensures that a pair of flavoured quarks originating from a wide-angle gluon splitting is clustered into a pseudo-jet before being combined with any other (harder) pseudo-jets. This avoids the situation where one of these soft quarks may be clustered with a hard pseudo-jet that carries zero flavour, which would lead to a definition of jet flavour sensitive to soft physics.

It is worth mentioning that, in our computation, we have chosen to define the flavour of pseudojets to be the net-flavour of its constituents modulo two, which means that all pseudo-jets which 
contain an even flavour content are considered to have zero net-flavour. The motivation for this choice is that, in our opinion, it is the most feasible realisation of the flavour- $k_{t}$ algorithm experimentally: Focussing on the case of b-jets, the main consideration is that most experimental approaches to flavour tagging are sensitive only to the absolute flavour [11-13] (and do not additionally charge tag the jets). All implementations of the algorithm must consider the combination of a b $\bar{b}$-quark pair (or equivalently a $\mathrm{B} \overline{\mathrm{B}}$-hadron pair) as carrying zero flavour, as required to guarantee its infrared safety as discussed above. Therefore, in the absence of charge tagging, any (pseudo)-jet which contains the presence of an even number of $b(B)$ and/or $\bar{b}(\bar{B})$ quarks (hadrons) should also be considered to carry zero flavour, as experimentally these signatures are indistinguishable.

\section{Ingredients of the computation}

In this section we present the main ingredients that enter the calculation of the Higgs Strahlung process at NNLO. We establish how those building blocks are assembled to express the cross section in a factorised form in terms of production and decay sub-processes.

\subsection{General framework}

We consider the process $\mathrm{pp} \rightarrow V \mathrm{H}+X \rightarrow \ell \bar{\ell} \mathrm{b} \overline{\mathrm{b}}+X$ where the vector boson $(V)$ decays leptonically and the Higgs boson $(\mathrm{H})$ decays into a pair of bottom quarks $b \bar{b}$. We compute NNLO QCD observables related to these reactions by including corrections up to order $\alpha_{\mathrm{s}}^{2}$ in both production and decay sub-processes. This enables us to express the fully differential cross section at the $k$ th order in a factorised form given as

$$
\mathrm{d} \sigma^{\mathrm{N}^{k} \mathrm{LO}}=\sum_{\substack{i, j=0 \\ i+j \leq k}}^{k} \mathrm{~d} \sigma_{V \mathrm{H}}^{(i)} \times \mathrm{d} \sigma_{\mathrm{H} \rightarrow \mathrm{b} \overline{\mathrm{b}}}^{(j)} .
$$

The term $\mathrm{d} \sigma_{V \mathrm{H}}^{(i)}$, which corresponds to the production part, comprises the vector propagator and the leptonic decay $V \rightarrow \ell \bar{\ell}$, including spin correlations between the initial-state partons and the finalstate leptons. The term denoted by $\sigma_{\mathrm{H} \rightarrow \mathrm{b} \overline{\mathrm{b}}}^{(j)}$ corresponds to the decay part and includes the Higgs propagator and its subsequent decay to a bottom-antibottom quark pair. We treat all light quarks as massless including the bottom quark with the exception of the Yukawa coupling mediating the $\mathrm{H} \rightarrow \mathrm{b} \overline{\mathrm{b}}$ decay. In the decay the bottom quark Yukawa coupling to the Higgs boson is renormalised in the $\overline{\mathrm{MS}}$ scheme at the scale $\mu^{\text {dec. }}$, taken to be proportional to the Higgs-boson mass $m_{\mathrm{H}}{ }^{1}$

At order $\mathscr{O}\left(\alpha_{\mathrm{s}}^{2}\right)$, the cross section may then be written as

$$
\begin{aligned}
\mathrm{d} \sigma^{\mathrm{NNLO}} & =\mathrm{d} \sigma_{V \mathrm{H}}^{(0)} \times\left(\mathrm{d} \sigma_{\mathrm{H} \rightarrow \mathrm{b} \overline{\mathrm{b}}}^{(0)}+\mathrm{d} \sigma_{\mathrm{H} \rightarrow \mathrm{b} \overline{\mathrm{b}}}^{(1)}+\mathrm{d} \sigma_{\mathrm{H} \rightarrow \mathrm{b} \overline{\mathrm{b}}}^{(2)}\right) \\
& +\mathrm{d} \sigma_{V \mathrm{H}}^{(1)} \times\left(\mathrm{d} \sigma_{\mathrm{H} \rightarrow \mathrm{b} \overline{\mathrm{b}}}^{(0)}+\mathrm{d} \sigma_{\mathrm{H} \rightarrow \mathrm{b} \overline{\mathrm{b}}}^{(1)}\right) \\
& +\mathrm{d} \sigma_{V \mathrm{H}}^{(2)} \times\left(\mathrm{d} \sigma_{\mathrm{H} \rightarrow \mathrm{b} \overline{\mathrm{b}}}^{(0)}\right) .
\end{aligned}
$$

\footnotetext{
${ }^{1}$ It is known from the computation of the inclusive cross section that this choice of regularisation scheme leads to a reduction of the size of the QCD corrections.
} 
Based on this master formula (3.2) for the $V H$ process at NNLO, we next specify the individual ingredients of the production and decay parts in the following and describe how they are assembled to the final prediction for the Higgs Strahlung process.

\subsection{Production parts}

Up to order $\alpha_{\mathrm{s}}$, only one type of contribution enters the associated Higgs production cross section, which is given by Drell-Yan-like diagrams with a subsequent Higgs emission from the gaugeboson leg. Starting from $\mathscr{O}\left(\alpha_{\mathrm{s}}^{2}\right)$, additional quark-loop induced contributions arise. These can be treated independently from the aforementioned Drell-Yan-type ones as the relevant Feynman amplitudes are individually gauge invariant. In the following, we describe these two production modes one after the other.

Drell-Yan-type: These contributions arise from the Drell-Yan-like production of a virtual $\mathrm{W}^{ \pm}$ or $\mathrm{Z}$ boson, which then splits into a real vector boson and a Higgs particle. In our calculation we include them up to $\mathscr{O}\left(\alpha_{\mathrm{s}}^{2}\right)$ using off-shell amplitudes that effectively treat both the directly produced vector boson and the vector boson that decays leptonically as virtual particles. These contributions only involve the square of Drell-Yan-like amplitudes and the infrared singularities are dealt with using the NNLO antenna subtraction formalism [14]. The subtraction terms can be readily constructed based on the NNLO calculation for the Drell-Yan processes, which are available within the NNLOJET framework.

Top-quark-loop induced: Starting from $\mathscr{O}\left(\alpha_{\mathrm{s}}^{2}\right)$, new types of diagrams induced by quark loops must be taken into account for the $V H$ production process. The contribution to the cross section either arises through the square of these loop-amplitudes or though the interference with Drell-Yantype amplitudes. Those included in our calculation are mentioned explicitly in [4]. We include in particular the amplitudes induced by gluon-gluon initial states, which are exclusively present in ZH production. Phenomenologically, they represent the dominant component among the toploop-induced contributions due to the large gluon luminosity at the LHC and were also considered in the previous calculations at NNLO. Note that all quark-loop-induced contributions are both infrared and ultraviolet finite and thus no subtraction procedure is needed in their evaluation. The heavy-quark-loop-induced contributions included in our calculation have been either independently rederived or implemented using known results. A validation of the implementation was performed against OpenLoops amplitudes [15] and full agreement was found in all cases.

\subsection{Decay parts}

For the decay sub-process $\mathrm{H} \rightarrow \mathrm{b} \overline{\mathrm{b}}$, we required the corrections up to $\mathscr{O}\left(\alpha_{\mathrm{s}}^{2}\right)$ as indicated in our master formula (3.2). The corresponding amplitudes at one- and two-loop level were obtained from the analytic expressions of refs. $[16,17]$ and were decomposed into different colour levels according to antenna formalism conventions. A validation of all one-loop amplitudes was performed against the OpenLoops library [15], yielding full agreement. In addition, subtraction terms capturing the infrared singularities are required. Those have been constructed for the Higgs decay up to order $\mathscr{O}\left(\alpha_{\mathrm{s}}^{2}\right)$ for the present computation. Checks for the correct divergent behaviour in all singleand double-unresolved limits have been performed in order to ensure the proper cancellation of 
singularities in the real-emission corrections as well as the cancellation of poles against the virtual amplitudes. The decay sub-process up to NNLO only enters in eq. (3.2) when combined with the Drell-Yan-type production parts. For the top-quark-loop induced contributions, which are already of $\mathscr{O}\left(\alpha_{\mathrm{s}}^{2}\right)$, the decay only needs to be considered at tree level.

\section{Numerical results}

It is the purpose of this section to present some of the main phenomenological results related to the computation of the Higgsstrahlung process at order $\alpha_{s}^{2}$ and presented in [4].

\subsection{General setup}

We provide predictions for proton-proton collisions at $\sqrt{s}=13 \mathrm{TeV}$ using the parton distribution function NNPDF31_nnlo_as_0118 provided via the LHAPDF library [18]. Each event is required to contain at least two b-jets with transverse momentum $p_{\perp, \mathrm{b}}>25 \mathrm{GeV}$ and rapidity $\left|y_{\mathrm{b}}\right|<2.5$. Charged leptons are required to have a transverse momentum above $p_{\perp, \ell}>15 \mathrm{GeV}$ and for their rapidity to satisfy $\left|y_{\ell}\right|<2.5$. For the $\mathrm{W}^{ \pm} \mathrm{H}$ processes, we additionally demand a minimum missing transverse energy of $E_{\perp \text {,miss }}>15 \mathrm{GeV}$ to identify the neutrino in the final state. We use the flavour- $k_{t}$ algorithm with an even-tag exclusion to cluster b-jets with the parameters $R=0.5$ and $\alpha=2$.

For the unphysical scales appearing in the calculation, we choose to set and vary them independently for the production and decay parts. The central factorisation and renormalisation scales of the production sub-processes are chosen to be the invariant mass of the $V \mathrm{H}$ system $M_{V \mathrm{H}}$, whereas the central renormalisation scale of the decay are set to the Higgs-boson mass $m_{\mathrm{H}}$. We evaluate the differential cross section for a total of 21 different scale settings that are obtained from all possible combinations of

$$
\mu_{\mathrm{F}}=M_{V \mathrm{H}}\left[1, \frac{1}{2}, 2\right], \quad \quad \mu_{\mathrm{R}}^{\text {prod. }}=M_{V \mathrm{H}}\left[1, \frac{1}{2}, 2\right], \quad \mu_{\mathrm{R}}^{\text {dec. }}=m_{\mathrm{H}}\left[1, \frac{1}{2}, 2\right],
$$

with the additional constraint $\frac{1}{2} \leq \mu_{\mathrm{F}} / \mu_{\mathrm{R}}^{\text {prod. }} \leq 2$ following the conventional 7-point scale variation for the production sub-process.

\subsection{Scale variations}

The dependence on the renormalisation scales $\mu_{\mathrm{R}}^{\text {prod./dec. }}$ can serve as a non-trivial check of the final results obtained from the numerical computation. To this end, we ensure that the different scale settings of eq. (4.1) are correctly reproduced by the analytic renormalisation-group running starting from the central scale choice. ${ }^{2}$ This is of particular importance for the calculation at hand, as the independent variation of scales for the different sub-processes was for the first time implemented in the NNLOJET framework for the present work.

\subsection{Fiducial cross sections}

In Table 1, we present the predictions including fiducial cuts for the different $V \mathrm{H}$ processes at the various orders in $\alpha_{\mathrm{s}}$ and using two different formulations of the cross section.

\footnotetext{
${ }^{2}$ For processes involving just a production part, the analytic expressions have been explicitly given in ref. [19].
} 
The first part of this table contains the cross section, denoted as unscaled and given as in eq. 3.1, while in the second half of this table a scaled variant of the cross section incorporating the Higgs-boson branching ratio at a fixed value and thus not subject to an $\alpha_{\mathrm{s}}$ expansion is tabulated. Up to NNLO, the cross section in this latter formulation is assembled as follows:

$$
\begin{aligned}
\mathrm{d} \sigma_{\mathrm{LO}}^{\text {scaled }} & =\mathrm{d} \sigma_{V \mathrm{H}}^{(0)} \times\left(\mathrm{d} \sigma_{\mathrm{H} \rightarrow \mathrm{b} \overline{\mathrm{b}}}^{(0)}\right) \times K^{(0)}, \\
\mathrm{d} \sigma_{\mathrm{NLO}}^{\text {scaled }} & =\mathrm{d} \sigma_{V \mathrm{H}}^{(0)} \times\left(\mathrm{d} \sigma_{\mathrm{H} \rightarrow \mathrm{b} \overline{\mathrm{b}}}^{(0)}+\mathrm{d} \sigma_{\mathrm{H} \rightarrow \mathrm{b} \overline{\mathrm{b}}}^{(1)}\right) \times K^{(1)} \\
& +\mathrm{d} \sigma_{V \mathrm{H}}^{(1)} \times\left(\mathrm{d} \sigma_{\mathrm{H} \rightarrow \mathrm{b} \overline{\mathrm{b}}}^{(0)}\right) \times K^{(0)}, \\
\mathrm{d} \sigma_{\mathrm{NNLO}}^{\text {scaled }} & =\mathrm{d} \sigma_{V \mathrm{H}}^{(0)} \times\left(\mathrm{d} \sigma_{\mathrm{H} \rightarrow \mathrm{b} \overline{\mathrm{b}}}^{(0)}+\mathrm{d} \sigma_{\mathrm{H} \rightarrow \mathrm{b} \overline{\mathrm{b}}}^{(1)}+\mathrm{d} \sigma_{\mathrm{H} \rightarrow \mathrm{b} \overline{\mathrm{b}}}^{(2)}\right) \times K^{(2)} \\
& +\mathrm{d} \sigma_{V \mathrm{H}}^{(1)} \times\left(\mathrm{d} \sigma_{\mathrm{H} \rightarrow \mathrm{b} \overline{\mathrm{b}}}^{(0)}+\mathrm{d} \sigma_{\mathrm{H} \rightarrow \mathrm{b} \overline{\mathrm{b}}}^{(1)}\right) \times K^{(1)} \\
& +\mathrm{d} \sigma_{V \mathrm{H}}^{(2)} \times\left(\mathrm{d} \sigma_{\mathrm{H} \rightarrow \mathrm{b} \overline{\mathrm{b}}}^{(0)}\right) \times K^{(0)} .
\end{aligned}
$$

Here, the scaling factors $K^{(i)}$ contain the branching ratio and are given by

$$
K^{(i)}=\frac{\mathrm{Br}(\mathrm{H} \rightarrow \mathrm{b} \overline{\mathrm{b}}) \Gamma_{\mathrm{H}}}{\sum_{j=0}^{i} \Gamma_{\mathrm{H} \rightarrow \mathrm{b} \overline{\mathrm{b}}}^{(j)}} .
$$

It is worth mentioning that this scaled variant of the cross section was essential in describing the data using fixed-order predictions at LO and NLO levels. With this formulation, the LO predictions have the correct normalisation; NLO corrections are kept small and have a small residual theoretical uncertainty. If computed up to order $\alpha_{\mathrm{s}}^{2}$, we here argue that the need of such scaling factors in the formulation of the cross section becomes questionable. Indeed, focussing on the NNLO results, we here see that both prescriptions agree well in their respective central values, however, sizeable differences can be seen in their associated uncertainties. The scaled predictions at NNLO show almost no reduction in scale uncertainties - even increasing for $\mathrm{ZH}$ - compared to the respective NLO number, whereas our formulation given in eq. 3.1 exhibits a substantial reduction in scale uncertainties when going from NLO to NNLO. This difference can be attributed to the compensation of scale dependences in the latter case, which is spoiled in the scaled formulation of this rate, by the different rescaling factors used in eq. (4.4) among different terms.

We therefore, find that a consistent treatment of theoretical uncertainties outweighs the precision gain that one might obtain by scaling the cross section to a fixed branching ratio, if the cross section is computed including NNLO corrections in both production and decay parts. This further motivates the simpler formulation of the cross section given above in eq. (3.2) where no scaling factors are applied. This set up has been used to present the results for distributions in [4] and in the present report in section 4.4.

\subsection{Distributions}

In [4] we present differential distributions of flavour-sensitive observables for the three different associated Higgs boson production processes $\mathrm{W}^{+} \mathrm{H}, \mathrm{W}^{-} \mathrm{H}$, and $\mathrm{ZH}$. Those are: (a) the transverse momentum $p_{\perp, \mathrm{b}}$ of the leading b-jet, (b) the transverse momentum $p_{\perp, \mathrm{bb}}$ of the pair of 


\begin{tabular}{r|ccc|ccc}
\multicolumn{3}{c}{ naïve (unscaled) } & \multicolumn{3}{c}{ scaled } \\
& $\mathrm{W}^{+} \mathrm{H}$ & $\mathrm{W}^{-} \mathrm{H}$ & $\mathrm{ZH}$ & $\mathrm{W}^{+} \mathrm{H}$ & $\mathrm{W}^{-} \mathrm{H}$ & $\mathrm{ZH}$ \\
\hline$\sigma_{\mathrm{LO}}[\mathrm{fb}]$ & $18.06_{-2.41}^{+2.87}$ & $11.96_{-1.60}^{+1.90}$ & $4.83_{-0.65}^{+0.77}$ & $22.52_{-0.80}^{+0.63}$ & $14.91_{-0.54}^{+0.42}$ & $6.02_{-0.21}^{+0.17}$ \\
$\sigma_{\mathrm{NLO}}[\mathrm{fb}]$ & $21.52_{-1.08}^{+0.88}$ & $14.21_{-0.71}^{+0.58}$ & $5.71_{-0.28}^{+0.22}$ & $22.87_{-0.87}^{+0.76}$ & $15.11_{-0.58}^{+0.51}$ & $6.06_{-0.23}^{+0.20}$ \\
$\sigma_{\mathrm{NNLO}}[\mathrm{fb}]$ & $20.68_{-0.46}^{+0.16}$ & $13.64_{-0.31}^{+0.11}$ & $5.92_{-0.16}^{+0.13}$ & $20.93_{-0.73}^{+0.61}$ & $13.80_{-0.49}^{+0.41}$ & $6.10_{-0.31}^{+0.31}$
\end{tabular}

two b-jets, (c) the angular separation $\Delta R_{\mathrm{bb}}=\sqrt{\Delta \eta_{\mathrm{bb}}^{2}+\Delta \phi_{\mathrm{bb}}^{2}}$ of two b-jets, and the invariant mass $m_{\mathrm{bb}}$ of two b-jets, where in (b-d) the two b-jets are selected whose invariant mass is closest to $m_{\mathrm{H}}$ in order to identify the candidate pair that is most likely to originate from the Higgs decay.

In this talk, we focus on presenting results concerning the transverse momentum $p_{\perp, \mathrm{bb}}$ of the pair of two b-jets for $W^{+} H$ and $Z H$ processes. We choose to show this distribution since it is one of the distribution where at NNLO the phenomenological differences between these two processes are the most pronounced. The results are given in Figure 1.

As can be seen, NNLO corrections to the $\mathrm{W}^{+} \mathrm{H}$ case lead to a substantial stabilisation of the prediction. Size and shape are only slightly modified at NNLO compared to the NLO predictions; the scale-variation bands, however, are reduced considerably. In contrast, the ZH distribution show an excess of events in the central region. This behaviour is attributed to top-quark-loop threshold effects in the dominant top-quark loop gluon-gluon-induced $\mathrm{ZH}$-exclusive amplitudes. As mentioned earlier, these channels first contributed at NNLO, which also explains the widening of the theoretical uncertainty bands around the threshold regions of these distributions.

\section{Conclusions and outlook}

In this talk, we summarised the main outcomes related to the computation of NNLO corrections to associated Higgs production observables presented in [4].

A detailed account was given on the residual theory uncertainties by allowing the scales in the production and decay sub-processes to vary independently. The NNLO corrections to the fiducial cross section were found to exhibit a good perturbative convergence with residual uncertainties at the percent level. We contrasted our naïve perturbative expansion of the cross section with a commonly employed rescaling procedure using the branching ratio $\mathrm{BR}(\mathrm{H} \rightarrow \mathrm{b} \overline{\mathrm{b}})$, where we observed that the residual scale uncertainties did not get considerably reduced, instead.

Flavour-sensitive observables were studied by investigating differential distributions where a similar stabilisation of the perturbative series was found as in the cross sections. A comparison between the $\mathrm{W}^{ \pm} \mathrm{H}$ and $\mathrm{ZH}$ processes emphasised the phenomenologically sizeable impact that arise from the gluon-gluon-induced top-quark loop amplitudes.

The study of flavour-sensitive jet observables with fixed-order predictions, must be performed in an infrared-safe way. For calculations based on massless QCD this can only be achieved with a flavour-aware jet algorithm (such as flavour- $k_{t}$ ). Future comparisons to measurements are only viable if a similar prescription is also employed in the experiment, and the application of the even-tag 


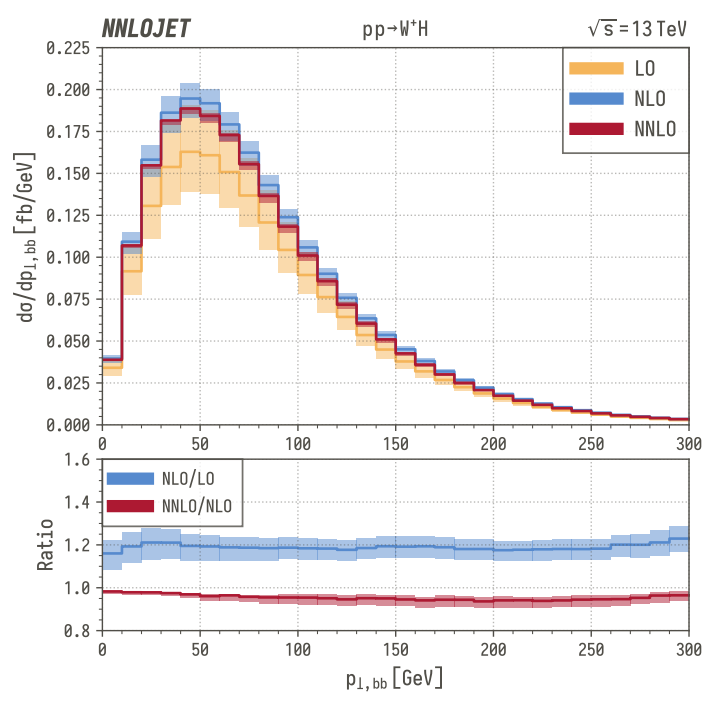

(a)

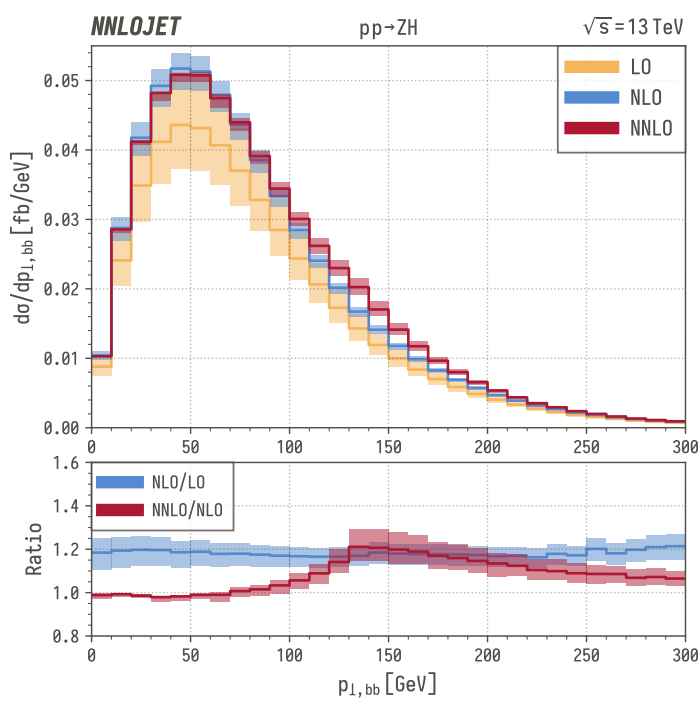

(b)

Figure 1: Flavour-sensitive jet distributions for the (a) $\mathrm{W}^{+} \mathrm{H}$ and (b) $\mathrm{ZH}$ processes showing the transverse momentum of the b-jet pair. The upper panel contains the absolute values while the lower panel shows the bin-by-bin ratios with respect to the previous order evaluated at the central scale.

exclusion here was mainly motivated to facilitate the experimental implementation. Such studies are left for future work.

\section{Acknowledgments}

This research was supported in part by the UK Science and Technology Facilities Council under contract ST/G000905/1, by the Swiss National Science Foundation (SNF) under contract 200021-172478, and by the Dutch Organisation for Scientific Research (NWO) through the VENI grant 680-47-461.

\section{References}

[1] ATLAS Collaboration, M. Aaboud et al., Observation of $H \rightarrow b \bar{b}$ decays and $V H$ production with the ATLAS detector, Phys. Lett. B786 (2018) 59-86, [arXiv: 1808 . 08238].

[2] CMS Collaboration, A. M. Sirunyan et al., Observation of Higgs boson decay to bottom quarks, Phys. Rev. Lett. 121 (2018), no. 12 121801, [arXiv: 1808.08242 ].

[3] ATLAS Collaboration, M. Aaboud et al., Measurement of $V H, \mathrm{H} \rightarrow \mathrm{b} \overline{\mathrm{b}}$ production as a function of the vector-boson transverse momentum in $13 \mathrm{TeV}$ pp collisions with the ATLAS detector, JHEP 05 (2019) 141, [arXiv:1903.04618]. 
[4] R. Gauld, A. Gehrmann-De Ridder, E. W. N. Glover, A. Huss, and I. Majer, Associated production of a Higgs boson decaying into bottom quarks and a weak vector boson decaying leptonically at NNLO in QCD, JHEP 10 (2019) 002, [arXiv:1907.05836].

[5] G. Ferrera, G. Somogyi, and F. Tramontano, Associated production of a Higgs boson decaying into bottom quarks at the LHC in full NNLO QCD, Phys. Lett. B780 (2018) 346-351, [arXiv:1705.10304].

[6] F. Caola, G. Luisoni, K. Melnikov, and R. Röntsch, NNLO QCD corrections to associated WH production and $H \rightarrow b \bar{b}$ decay, Phys. Rev. D97 (2018), no. 7 074022, [arXiv: 1712.06954 ].

[7] A. Banfi, G. P. Salam, and G. Zanderighi, Infrared safe definition of jet flavor, Eur. Phys. J. C47 (2006) 113-124, [hep-ph/0601139].

[8] J. Currie, E. W. N. Glover, and S. Wells, Infrared Structure at NNLO Using Antenna Subtraction, JHEP 04 (2013) 066, [arXiv: 1301 . 4693].

[9] A. Banfi, G. P. Salam, and G. Zanderighi, Accurate QCD predictions for heavy-quark jets at the Tevatron and LHC, JHEP 07 (2007) 026, [arXiv: 0704.2999 ].

[10] M. Cacciari, G. P. Salam, and G. Soyez, The anti-kt jet clustering algorithm, JHEP 04 (2008) 063, [arXiv:0802.1189].

[11] LHCb Collaboration, R. Aaij et al., Identification of beauty and charm quark jets at LHCb, JINST 10 (2015), no. 06 P06013, [arXiv: 1504 .07670].

[12] ATLAS Collaboration, G. Aad et al., Performance of b-Jet Identification in the ATLAS Experiment, JINST 11 (2016), no. 04 P04008, [arXiv: 1512.01094 ].

[13] CMS Collaboration, A. M. Sirunyan et al., Identification of heavy-flavour jets with the CMS detector in pp collisions at $13 \mathrm{TeV}$, JINST 13 (2018), no. 05 P05011, [arXiv: 1712. 07158].

[14] A. Gehrmann-De Ridder, T. Gehrmann, and E. W. N. Glover, Antenna subtraction at NNLO, JHEP 09 (2005) 056, [hep-ph/ 0505111$].$

[15] F. Cascioli, P. Maierhöfer, and S. Pozzorini, Scattering Amplitudes with Open Loops, Phys. Rev. Lett. 108 (2012) 111601, [arXiv:1111.5206].

[16] C. Anastasiou, F. Herzog, and A. Lazopoulos, The fully differential decay rate of a Higgs boson to bottom-quarks at NNLO in QCD, JHEP 03 (2012) 035, [arXiv: 1110.2368 ].

[17] V. Del Duca, C. Duhr, G. Somogyi, F. Tramontano, and Z. Trócsányi, Higgs boson decay into b-quarks at NNLO accuracy, JHEP 04 (2015) 036, [arXiv: 1501 . 07226].

[18] A. Buckley, J. Ferrando, S. Lloyd, K. Nordström, B. Page, M. Rüfenacht, M. Schönherr, and G. Watt, LHAPDF6: parton density access in the LHC precision era, Eur. Phys. J. C75 (2015) 132, [arXiv:1412.7420].

[19] J. Currie, A. Gehrmann-De Ridder, T. Gehrmann, E. W. N. Glover, A. Huss, and J. Pires, Infrared sensitivity of single jet inclusive production at hadron colliders, JHEP 10 (2018) 155, [arXiv:1807.03692]. 